\title{
Mucinous epithelial neoplasms of the appendix and pseudomyxoma peritonei
}

\author{
Joseph Misdraji \\ Department of Pathology, Massachusetts General Hospital, Harvard Medical School, Boston, MA, USA
}

\begin{abstract}
The classification of appendiceal mucinous neoplasms has been controversial, largely focused on a particular subset of low-grade mucinous tumors that, despite their innocuous appearance, can disseminate to the peritoneal cavity as pseudomyxoma peritonei (PMP). Recent WHO classification of these tumors as low-grade appendiceal mucinous neoplasms acknowledges their unique morphologic appearance and biologic behavior. Still, debate about the use of this term and its parameters continues to impede the adoption of consensus classification for appendiceal mucinous neoplasms. The classification of PMP has also been the subject of debate, with international authorities advocating for the use of malignant terminology to describe all grades of PMP, even though some authorities consider low-grade PMP to be dissemination of adenomatous epithelium in the peritoneum. Recent data also emphasize the importance of histologic grade of the peritoneal tumors in defining prognosis of these patients.
\end{abstract}

Modern Pathology (2015) 28, S67-S79; doi:10.1038/modpathol.2014.129

Appendiceal mucinous tumors include a spectrum of tumors, but within that spectrum is a fascinating low-grade tumor that has been the subject of considerable debate. These tumors challenge our very definition of malignancy and force us to consider whether biology, morphology, or both should form the basis of tumor classification. Despite their rather innocuous gross and microscopic appearance, these low-grade tumors can penetrate into or through the appendix wall, rupture the appendix, and ultimately disseminate to the peritoneal cavity as pseudomyxoma peritonei (PMP; Figures 1 and 2). Biologically, then, these tumors behave as low-grade malignancies and as such are managed aggressively when they have disseminated widely in the peritoneum. Predicting which appendiceal tumors will disseminate to the peritoneum is often a challenge for pathologists faced with a lowgrade mucinous tumor in the appendix that has perforated. Because these low-grade appendiceal tumors have bland cytologic features, non-neoplastic conditions that result in reactive epithelial atypia and mucin extrusion are commonly misinterpreted as a low-grade appendiceal mucinous neoplasm (LAMN). In this article, I describe the classification,

Correspondence: Dr J Misdraji, MD, Department of Pathology, Massachusetts General Hospital, Harvard Medical School, 55 Fruit Street, Warren 831, Boston, MA 02114, USA.

E-mail: jmisdraji@partners.org

Received 13 June 2014; revised 4 August 2014; accepted 5 August 2014 biology, and management of mucinous appendiceal tumors with an emphasis on low-grade tumors. Their differential diagnosis, natural history, and management are reviewed. In addition, entities commonly mistaken for appendiceal neoplasia will be addressed. Finally, the pathology of PMP will be reviewed, with an emphasis on tumor grade and its prognostic implications.

\section{Historical perspective}

The phenomenon of PMP was originally described in 1884 by Werth, who attributed the abdominal mucin to an ovarian cyst. ${ }^{1}$ In 1901, Fraenkel $^{2}$ recognized pseudomyxoma in the setting of an appendiceal tumor, and provides the first account of the association of an appendiceal tumor with PMP. Still, for decades, there was uncertainty about the nature of pseudomyxoma and whether it was related to the appendix, the ovary, or primary in the peritoneum. ${ }^{3}$ Meanwhile, an 'appendiceal mucocele' was an oft-diagnosed condition even if there was confusion about what that broad category comprised. There were efforts to distinguish between a benign and malignant 'mucocele', with a growing appreciation for the fact that PMP was progressive only if the mucocele was malignant. For example, in 1940, Woodruff and McDonald ${ }^{4}$ classified 146 cystic tumors of the appendix into simple mucoceles or adenocarcinoma, grade 1 . In their classification, only those tumors that were classified as 


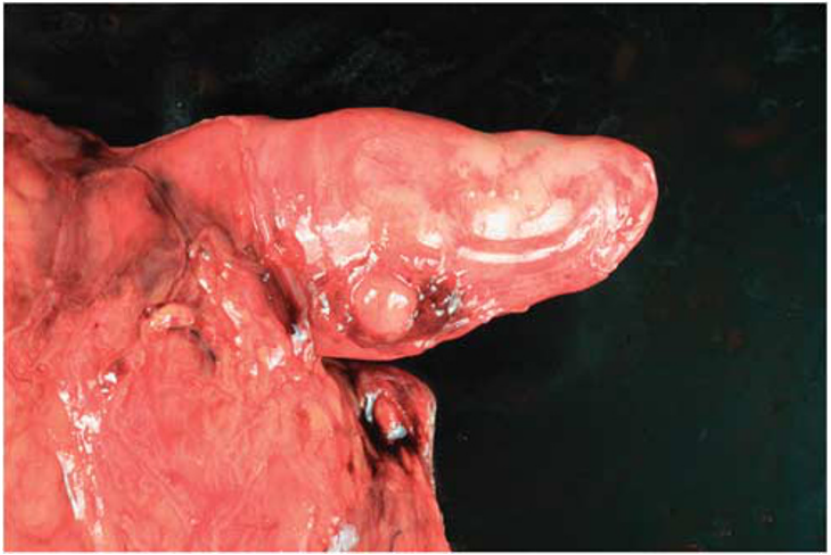

Figure 1 Gross appearance of a low-grade appendiceal mucinous neoplasm. The appendix is dilated and enlarged, although from the external appearance alone, the differential includes benign and malignant conditions that can cause a dilated appendix. The serosa shows a mucoid hemorrhagic plug that, while innocuous in appearance, is the point of egress of tumor epithelium into the peritoneum.

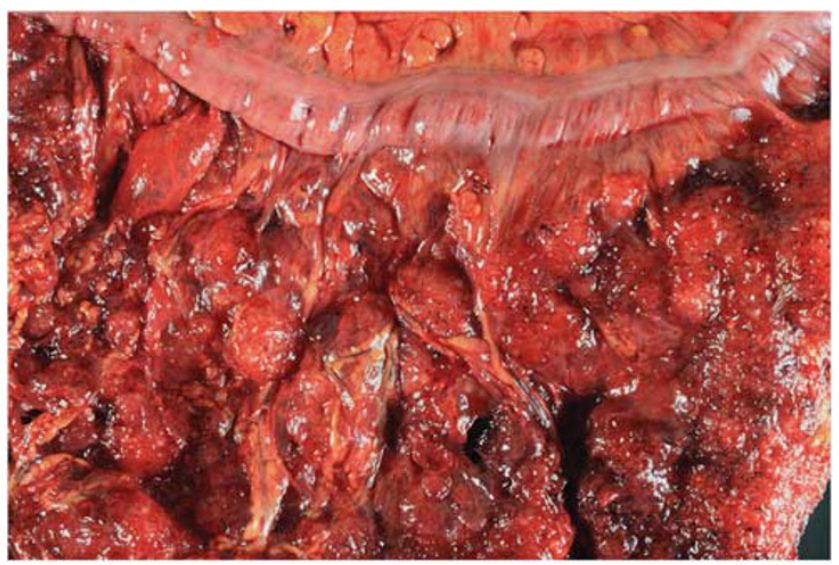

Figure 2 Gross appearance of the omentum from the patient with the appendix in Figure 1. The omentum shows adherent mucoid implants from the appendiceal tumor, producing the syndrome of pseudomyxoma peritonei.

adenocarcinoma could produce PMP. However, their images and description of tumors that they classified as adenocarcinoma show cytologic features that would be recognized by pathologists today as being similar to low-grade dysplasia. Appendiceal grade 1 adenocarcinomas were described as having 'papillary arrangements of the mucous membrane' with nuclei that are 'hyperchromatic and tend to extend up the length of the cell rather than limit themselves to the lower part.'4 Over the ensuing decades, various writers questioned whether these tumors were in fact malignant, as ones that are confined to the appendix are cured by appendectomy. Terminology for colon polyps was extrapolated to the appendix, and the concept of an appendiceal cystadenoma or villous adenoma became widely accepted, with some authors even using these names for tumors associated with PMP. However, the use of benign terminology for tumors that had disseminated led to the controversy of whether pseudomyxoma, essentially the metastasis of the appendiceal tumor, is benign or malignant. Is a low-grade cystic appendiceal mucinous tumor that ruptures and disseminates to the peritoneum a ruptured 'adenoma'? Several authorities, ${ }^{5-10}$ noting that the epithelium in the peritoneal metastasis is remarkably bland and that the tumor in the appendix shares features with an appendiceal adenoma, accepted that adenomatous epitheliumbeing neoplastic-might disseminate in the peritoneal cavity if given access to the peritoneum. Morphologic assessments also noted that these low-grade tumors are often associated with diverticula, herniations, dissection, or frank rupture, any of which could provide a route by which neoplastic epithelium disseminates to the peritoneum. Others take issue with labeling any tumor that has disseminated to the peritoneum as adenoma, or suggesting that the intraperitoneal mucinous epithelium is 'benign'. These authorities, including the American Joint Committee on Cancer and the World Health Organization, consider peritoneal dissemination to be evidence of malignancy and view lowgrade mucinous tumors that disseminate as welldifferentiated adenocarcinomas. ${ }^{11-15}$ They point out that mucinous tumors elsewhere in the body can be deceptively bland and that the concept of a ruptured adenoma does not account for the progressive behavior of the tumor once it spreads to the peritoneum. According to this view, these tumors are in fact invasive, but rather than usual infiltrative pattern, the invasion is 'pushing' or 'expansile' invasion, which pathologists have difficulty recognizing as invasion.

Because the criteria for pushing invasion in these tumors are not easily defined, and given the historical developments in the classification of these tumors, it is perhaps not surprising that various names have been proposed for the subset of tumors that, despite not having infiltrative invasion, disseminate to the peritoneum. Some years ago, the term mucinous tumor of uncertain malignant potential was proposed for these tumors. ${ }^{16}$ Subsequently, we proposed the term LAMN ${ }^{17}$ in order to account for their indolent but progressive malignant behavior yet absence of infiltrative invasion or highgrade cytology. More recently, Pai et al ${ }^{18}$ suggested dividing LAMNs into those that are at low risk of recurrence or at high risk of recurrence based on whether they were associated with acellular or cellular mucinous ascites, respectively. In the most recent WHO classification of appendiceal tumors, the terminology of LAMN was incorporated into the spectrum of appendiceal adenocarcinomas as a lowgrade mucinous adenocarcinoma with pushing rather than infiltrative invasion (Table 1). ${ }^{14}$ Yet this 
classification is recent and still not fully accepted. Next, as we review appendiceal mucinous neoplasms, we must recognize that the historical confusion in the nomenclature of appendiceal mucinous neoplasms limits our ability to distill from the literature the distinguishing clinical features of adenomas, LAMNs, and mucinous adenocarcinomas.

\section{Adenoma}

\section{Clinical Features}

Adenomas typically occur in patients in their fifth decade but the age range is wide. ${ }^{7,9,12,19}$ There is a female predominance. ${ }^{7,9,12,19}$ Abdominal pain that mimics acute appendicitis is the most common clinical presentation. ${ }^{9,12}$ Less often, an appendiceal adenoma presents as an abdominal mass or as intussusception of the appendix. ${ }^{9,12,20-28}$ Approximately half of the cases are discovered in asymptomatic patients, particularly in women who have undergone an appendectomy during gynecologic surgery. Occasional patients with adenomas in the appendix have concomitant colonic adenocarcinoma. ${ }^{9,12,19}$ However, whether this represents merely chance association is uncertain. Appendiceal adenomas are benign and are treated by appendectomy alone.

\section{Pathology}

Grossly, an appendix with an adenoma may be either unremarkable or dilated with tenacious mucin. The serosa is typically smooth unless there are adhesions, but gross mucin on the serosa is absent. ${ }^{12}$ On microscopic examination, by definition, these tumors are confined to the mucosa, meaning that the muscularis mucosae is intact throughout the lesion and there is no mucin dissecting into the wall or mucin extrusion outside the appendix. The tumor consists of a proliferation of mucinous epithelial cells, often with atrophy of the lymphoid tissue. The tumor is usually composed of filiform villi with scant lamina propria; the villi are lined by mucinous epithelial cells, often with abundant intracytoplasmic mucin (Figure 3). ${ }^{5,16,17}$ In some cases, mucin accumulation leads to cystic dilatation of the appendix. In these cases, one may see a flat, undulating, or short villous pattern of the neoplastic epithelium.

Unlike colonic adenomas, the majority of which are tubular adenomas, appendiceal adenomas are usually villous and circumferentially involve the appendix lumen. Localized tubular adenomas are rare in the appendix ${ }^{29,30}$ although they may occur in patients with familial adenomatous polyposis. ${ }^{11,16}$ The histologic appearance of these polyps is identical to those that occur in the colon. 


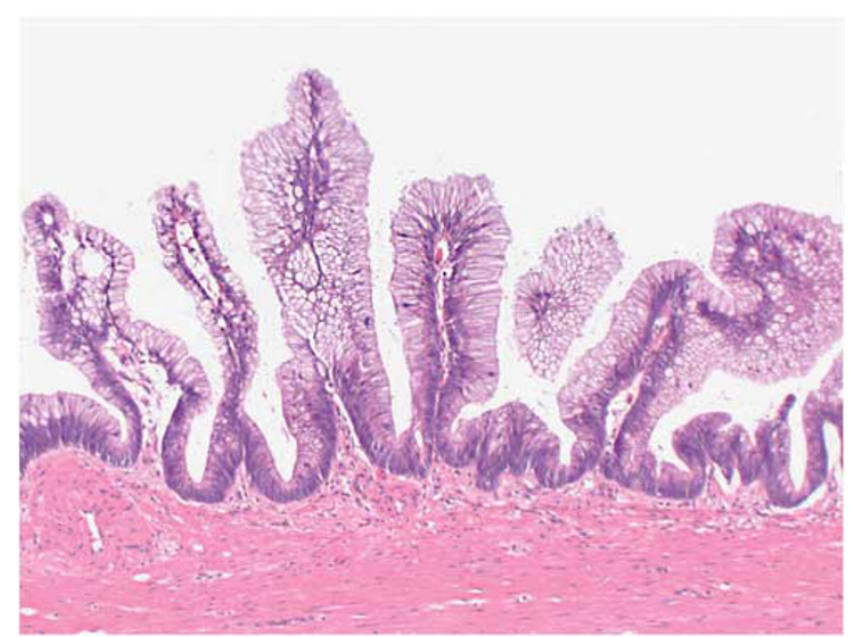

Figure 3 Appendiceal adenoma. The tumor shows replacement of the appendiceal mucosa by a villiform mucinous epithelial proliferation consisting of tall mucinous epithelial cells with low-grade nuclear atypia. Note the intact muscularis mucosae.

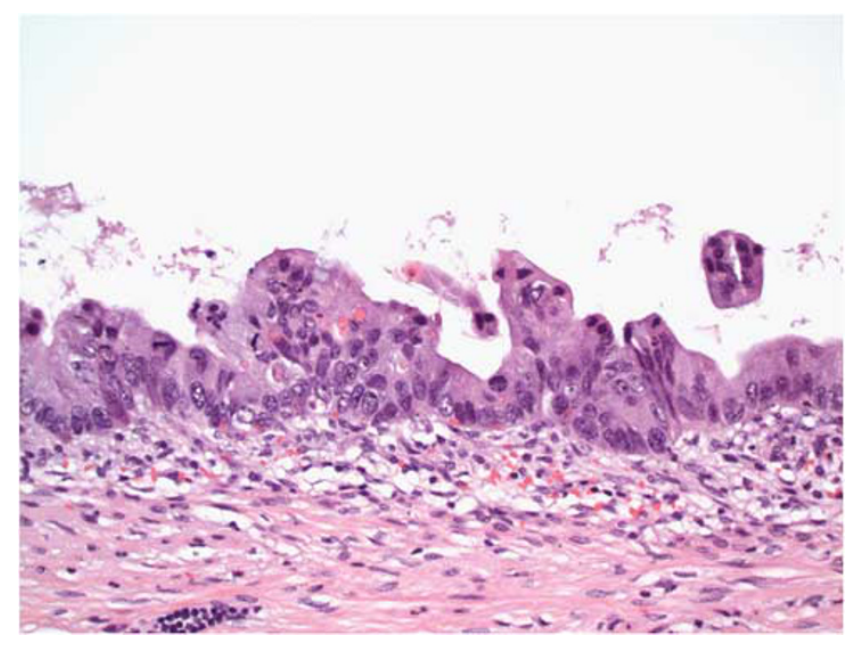

Figure 4 Appendiceal adenoma with high-grade dysplasia. In contrast to the tumor in Figure 3, this tumor shows pleomorphic and enlarged nuclei, with marked disarray and hyperchromasia. Note that the muscularis mucosae is intact.

Appendiceal adenomas are graded as either low or high, similar to colonic adenomas. Most tumors are low grade with nuclear elongation, hyperchromasia, pseudostratification, rare mitoses, and apoptotic nuclear debris. ${ }^{12,17,31}$ High-grade dysplasia shows greater atypia that is incompatible with low-grade dysplasia, such as micropapillary tufts with luminal necrosis, pleomorphic large nuclei, prominent nucleoli, and increased mitotic figures (Figure 4).

\section{Differential diagnosis}

The main diagnostic challenges are distinguishing cystic adenomas from retention cysts, and distinguishing adenomas from LAMNs. In cystic tumors, attenuation and denudation of the lining may make distinction with a retention cyst difficult. In this case, submitting the entire appendix may be necessary in order to detect the presence of neoplastic epithelium. The finding of nuclear pseudostratification in completely flat epithelium suggests that the lesion is neoplastic although reactive atypia can produce nuclear pseudostratification. More helpful is evaluation of the lining for residual focal papillary architecture together with the typical tall mucinous columnar cells. Distinction between an adenoma and a LAMN rests on evaluation of the muscularis mucosae that is intact throughout the entirety of an adenoma and is breached by dissecting mucin and/or epithelium in a LAMN (see LAMN).

\section{Low-grade appendiceal mucinous neoplasm}

\section{Clinical Features}

LAMNs usually present in the sixth decade. ${ }^{17,32}$ As with adenomas, there is a predilection for females. ${ }^{18}$ Presentation as an abdominal mass or as an ovarian metastasis is relatively common. ${ }^{12,17,32}$ Other presentations include abdominal pain or distention, possibly due to PMP. ${ }^{17,18}$ Rarely, a ruptured LAMN presents with mucin in a hernia sac. ${ }^{33-35}$ Approximately $15-20 \%$ of LAMNs are incidental findings in patients undergoing surgery for unrelated conditions. ${ }^{17,18}$

\section{Pathology}

Appendices with LAMNs may appear grossly unremarkable, or may be cystically dilated and filled with tenacious mucin. ${ }^{12,17,32,36}$ The wall may be thin or fibrotic, hyalinized, and calcified. ${ }^{12,17}$ Gross rupture may show mucin in the wall or on the serosal surface (Figure 1). ${ }^{12,17,32}$ The appendix lining may be smooth, granular, or corrugated with intervening smooth areas, similar to adenomas. ${ }^{12}$

On microscopic examination, these tumors show a villous or flat neoplastic mucinous epithelium often associated with atrophy of lymphoid tissue, similar to adenomas. ${ }^{5,16,17}$ In villous tumors, the villi are typically filiform and contain scant lamina propria. The epithelium often contains abundant cytoplasmic mucin that may compress nuclei that then may appear deceptively hyperplastic in nature rather than neoplastic. ${ }^{5}$ In uncommon cases, the cells resemble those in colonic type adenomas, with elongate pseudostratified nuclei, apical cytoplasm, and absent or reduced cytoplasmic mucin. Mucin accumulation within the lumen may be extensive and in these cystic tumors, the lining epithelium, which may be flat or undulating.

The defining feature of LAMN is the pattern of invasion of the underlying appendiceal wall that has been described as 'pushing' invasion and can have 


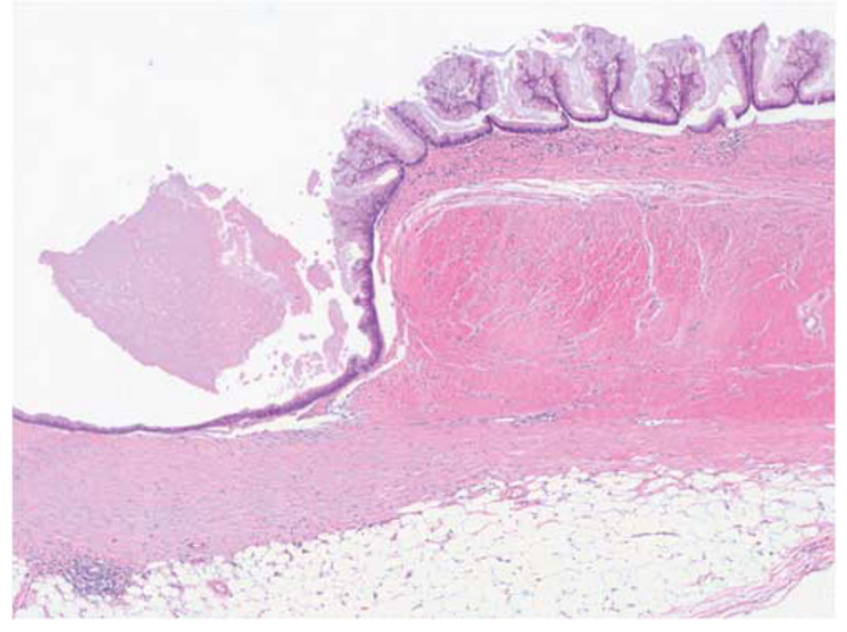

Figure 5 Pushing invasion in low-grade appendiceal mucinous neoplasm. In this tumor, the low-grade mucinous proliferation plunges through the muscularis propria, with complete absence of muscle along the left side of the field. Note that the tumor is bound on the left side of the field only by a small amount of fibrotic tissue and fat, rather than the full complement of appendiceal layers.

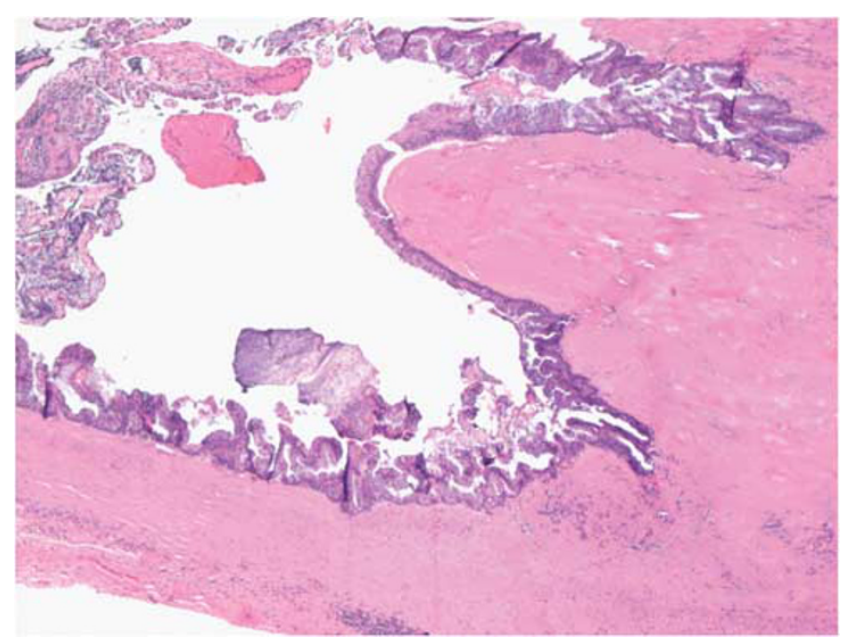

Figure 6 Pushing invasion in low-grade appendiceal mucinous neoplasm. In this example, the various layers of the appendix have been replaced by hyalinized fibrotic tissue. The low-grade mucinous epithelium grows along this hyalinized stroma rather than atop muscularis mucosae, consistent with pushing invasion. Note the absence of desmoplasia, tumor budding, or single cell invasion.

various patterns. The muscularis propria may be attenuated or even absent (Figure 5), but is often fibrotic or hyalinized. Neoplastic epithelium growing atop fibrotic or hyalinized stroma rather than lamina propria and muscularis mucosae is a frequent appearance of these tumors, and considered a sign of pushing invasion (Figure 6). Neoplastic epithelium may also push into or through the wall as diverticula, tongues of epithelium, or dissecting

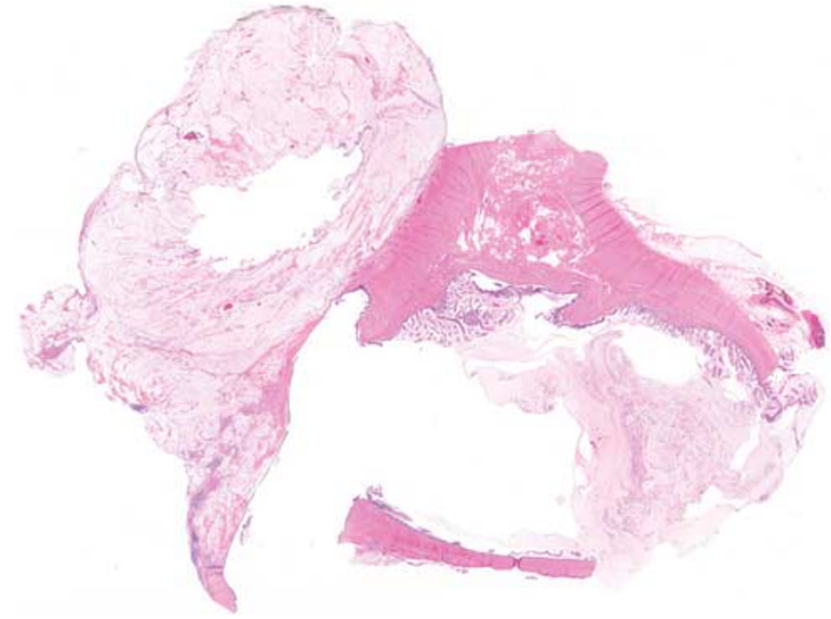

Figure 7 Rupture of a low-grade appendiceal mucinous neoplasm. The tumor pushes through the muscularis mucosae along the left wall of the appendix, with mucin extruding from the appendix and forming a rounded accumulation of mucin and fibrosis on the serosa (left).

epithelium, but usual features of infiltrative type invasion, such as single cell invasion, desmoplastic stromal response, or tumor budding are absent. The wall may be entirely breached, producing a picture of a ruptured cystic tumor with mucin extrusion onto the peritoneal surface, with or without mucinous epithelial cells growing freely in that mucin (Figure 7).

\section{Differential Diagnosis}

The main diagnostic challenge when evaluating a LAMN is deciding if other terminology is more appropriate, particularly adenoma or mucinous adenocarcinoma. Because they resemble adenomas in terms of their low-grade cytology and flat or villiform architecture, LAMNs that perforate the appendix might suggest that they are 'ruptured adenomas', but any tumor with mucin and/or epithelium dissecting within or outside the wall of the appendix should not be classified as an adenoma. If there is infiltrative invasion, of course, it qualifies as adenocarcinoma, but even without obvious infiltrative invasion, the possibility of pushing invasion must be considered, keeping in mind that epithelium pushing into the wall of the appendix, growing along hyalinized stroma, or perforating the appendix are considered by some authorities as evidence of pushing invasion by the tumor. When a tumor has disseminated to the peritoneal cavity, there still is debate about whether the appendix tumor should be classified as LAMN or as adenocarcinoma. However, the term LAMN reconciles the cytoarchitectural features of these tumors with their ability to spread to the peritoneum, and classifying these tumors as LAMN conforms to WHO recommendations. 


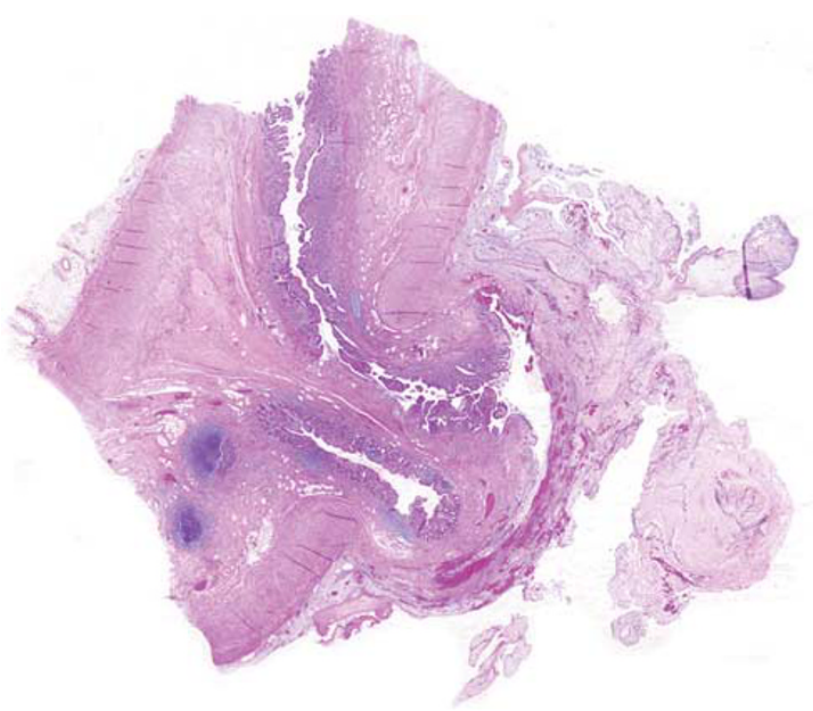

Figure 8 Appendiceal diverticular disease perforating the appendix. The diverticula penetrate the muscularis and result in mucin extrusion onto the serosal surface (right). Note the tangential sectioning and reactive changes that result in architectural disarray of the mucosa that can be mistaken for neoplasia.

Because LAMNs may have remarkably bland cytology and can push into or through the appendix without a desmoplastic response, benign processes can mimic LAMNs when they are associated with mucinous epithelial hyperplasia, dissection into the wall, appendiceal perforation, or mucin extrusion into the wall or onto the serosa. The most common mimic of LAMN is appendiceal diverticular disease that may, on occasion, rupture and cause extrusion of mucin into the wall or onto the appendiceal serosa (Figure 8). ${ }^{37}$ In addition, hyperplastic and reactive changes of the lining epithelium within the diverticula may be confused with a neoplastic process. An everted diverticulum, or fragments of diverticular lining on the serosa, may be confused with serosal involvement by LAMN. There are some features that differentiate ruptured diverticulum from a mucinous neoplasm. In patients with diverticular disease, the appendix may show several diverticula, and intact ones assist the pathologist in considering the possibility of perforated diverticular disease. Hyperplastic and reactive changes show architectural changes that are more pronounced in the superficial rather than the basal portions of the mucosa. Gland serration, crypt disarray, and hyperplastic changes with abundant mucin producing cells are usually located in the upper half of the mucosa. Non-neoplastic crypts are normally separated by lamina propria and show little or no crowding. In contrast, typical LAMNs show back-to-back crypts with scant lamina propria and elongated slender villi that protrude into the lumen of the appendix. One other helpful hint is that appendices with diverticula often show

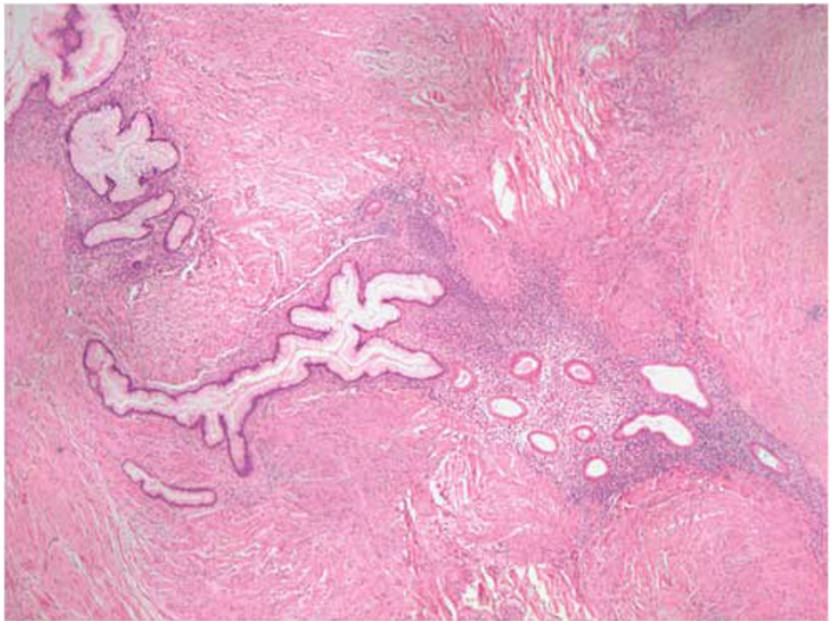

Figure 9 Endometriosis with intestinal metaplasia. The appendix shows an irregular cluster of glands surrounded by endometrial type stroma. Note that the glands on the right side of the field are endometrial type, whereas the glands on the left side of the field are composed of tall mucinous epithelial cells. The presence of mucinous glands dissecting in the wall can mimic a low-grade appendiceal mucinous neoplasm, particularly if the stomal component is scant.

Schwann cell proliferations that expand the lamina propria with a vaguely nodular proliferation of spindle cells, possibly because of the effects of obstruction. ${ }^{37,38}$

Appendiceal endometriosis with intestinal metaplasia represents a rare mimic of LAMN. ${ }^{39-43}$ Endometriosis with intestinal metaplasia can lead to mucin extrusion on the appendiceal serosa, causing confusion with a ruptured LAMN. Features that may suggest LAMN include extrusion of mucin in the mesoappendix or on the appendiceal serosa, marked distortion and fibrosis of the appendix, and intestinal type mucinous epithelium dissecting through the appendiceal muscularis propria. However, other features of these cases are helpful in distinguishing them from LAMN including absence of a definitive luminal mucinous neoplasm, intestinal glands in continuity with endometrial type glands, and a cuff of endometrial type stroma around the intestinal type glands (Figure 9), although the amount of stroma around individual glands can vary, and immunohistochemical markers of endometrial stroma may be useful to highlight scant stroma around some glands.

\section{Natural History}

The prognosis of LAMN is highly dependent on the stage of the tumor. When it is widely disseminated in the peritoneum, it pursues a progressive clinical course (see PMP). However, when the tumor is limited to the appendix or, when ruptured, confined to the right lower quadrant, the prognosis is related 
to the presence or absence of mucin and in particular of neoplastic mucinous epithelium outside the appendix. ${ }^{12,17,18}$ LAMNs that are confined to the appendix, without extra-appendiceal mucin, are essentially cured by appendectomy similar to adenomas. LAMNs associated with acellular mucin confined to the right lower quadrant carry a very low risk of recurrence or progression to PMP, whereas those associated with mucin and neoplastic mucinous epithelial cells in the right lower quadrant carry a relatively high risk of recurring as disseminated disease. In a multi-institutional study, ${ }^{44}$ the authors collected 65 patients with ruptured appendiceal mucinous tumors and at least 6 months of follow-up. Fifty of these patients had acellular mucin in the right lower quadrant, and only two $(4 \%)$ of them had recurrence. In contrast, 15 tumors were associated with clusters of lowgrade neoplastic epithelium within the extraappendiceal mucin. Five of these cases (33\%) recurred as diffuse PMP and one of these patients ultimately died of disease. In a series of 116 mucinous tumors, ${ }^{18} 12$ patients had acellular mucin in the right lower quadrant and 1 recurred. (Another two patients had acellular mucin beyond the RLQ and did not recur.) However, three of four $(75 \%)$ patients with extra-appendiceal mucin with neoplastic epithelium limited to the RLQ recurred. From these studies, it can be concluded that appendiceal mucinous tumors with acellular mucin in the right lower quadrant only rarely recur, whereas tumors with extra-appendiceal mucin containing neoplastic epithelium recur in about half of cases. Thus, in the evaluation of appendices with LAMNs, the entire specimen should be examined to evaluate for the presence of mucin on the serosa, and more importantly, the presence or absence of mucinous epithelium within the extra-appendiceal mucin.

\section{Treatment}

The treatment of a LAMN varies according to the stage of disease. Simple appendectomy is considered sufficient treatment for LAMNs that are confined to the appendix. In this case, a positive margin requires that a decision be made as to whether a cecectomy is necessary. Although there is limited data on this question, in our experience, resection of the cecum is not routinely performed, and residual tumor in the resection specimen is typically absent, indicating that conservative management may be an acceptable option. ${ }^{45}$

Once a LAMN ruptures, the risk of developing PMP is defined by whether acellular or cellular mucin is extruded. Patients with acellular mucin on the appendiceal serosa should be followed closely to ensure that PMP does not develop. Radiographic imaging of the abdomen and pelvis is often the preferred method of surveillance. Tumors that have spread to the right lower quadrant (mucin with epithelial cells present outside the appendix in the RLQ) run a higher risk of recurrence, but their management is not defined. Right hemicolectomy offers no additional benefit over appendectomy alone for these patients and is probably best done by a surgeon with expertise in the management of peritoneal malignancy who can arrange definitive cytoreductive surgery if necessary. ${ }^{46,47}$ Tumors that have disseminated widely throughout the peritoneal cavity were treated historically by debulking but, more recently, with cytoreductive surgery and hyperthermic intraperitoneal chemotherapy (HIPEC; see PMP).

\section{Adenocarcinoma}

\section{Clinical Features}

Adenocarcinomas of the appendix are uncommon; Collins et $a l^{48}$ found an incidence of $0.082 \%$ among 50000 appendectomy specimens. There is an increased incidence among men in some series ${ }^{8,49-56}$ but not in others. ${ }^{57-60}$ Patients are usually in their fifth to seventh decade of life at presentation ${ }^{49,51-53,55-58,60}$ and usually present with symptoms of acute appendicitis. Other less common modes of presentation include a palpable mass, obstruction, gastrointestinal bleeding, or symptoms related to metastases. ${ }^{7,8,51-54,56-59,61-63}$

\section{Pathology}

Appendiceal adenocarcinomas are classified as adenocarcinoma not otherwise specified, mucinous adenocarcinoma, signet ring cell adenocarcinoma, and undifferentiated carcinoma. ${ }^{14}$ Mucinous adenocarcinoma accounts for $\sim 40 \%$ of all appendiceal adenocarcinomas although it is unclear what proportion of these might be classified as LAMN according to current WHO terminology. ${ }^{52}$ The histology of invasive mucinous adenocarcinomas of the appendix is similar to those that occur elsewhere in the colon, with pools of mucin infiltrating the wall of the appendix harboring cytologically malignant glandular epithelium arranged as strips, clusters, or complex glandular structures (Figures 10). Mucinous adenocarcinomas may not only rupture and metastasize to the peritoneum, ${ }^{59,62}$ but can also spread via a hematogenous route. ${ }^{7,46,53,64}$ Grossly, peritoneal metastases may resemble those that occur in LAMNs. Histologically, the peritoneal tumor is usually more cellular, and the neoplastic epithelium shows a greater degree of architectural complexity and higher-grade cytologic atypia, desmoplastic stroma, and possibly signet ring cells (see PMP). Other organs may be affected such as small or large bowel, liver, or ovary. ${ }^{10,65}$

Non-mucinous appendiceal adenocarcinomas show a range of morphologic features of the invasive component. The tumors may resemble colonic 


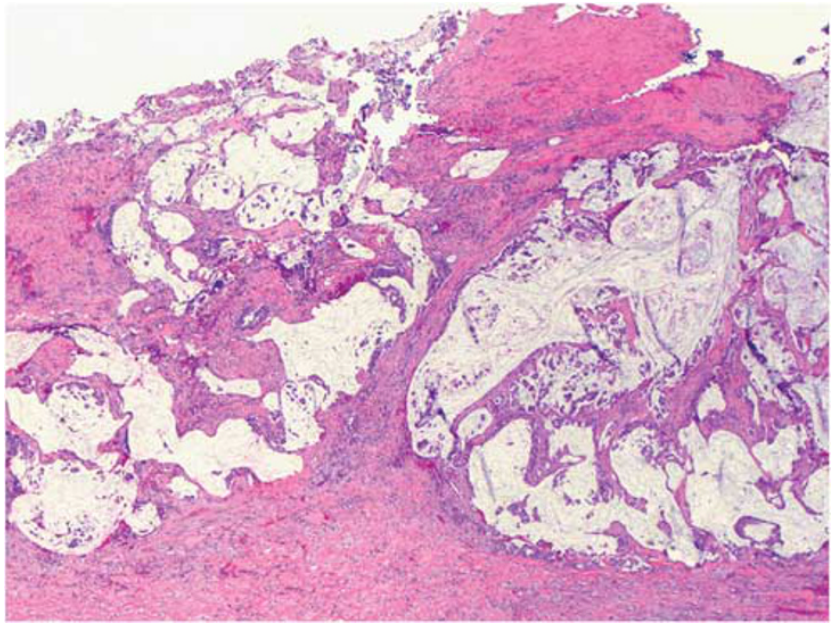

Figure 10 Invasive mucinous adenocarcinoma of the appendix. As opposed to low-grade appendiceal mucinous neoplasms that show pushing invasion, this tumor shows infiltrative invasion, with irregular mucin pools with numerous disordered buds of tumor in the mucin and desmoplasia.

adenocarcinomas with malignant glands lined by columnar cells with central dirty necrosis (Figure 11). Other tumors are composed of tubular glands lined by cuboidal cells, without dirty necrosis, and with only scant extracellular mucin, reminiscent of pancreaticobiliary adenocarcinomas.

Signet ring cell adenocarcinomas are rare in the appendix. These tumors are associated with a poor prognosis owing to rapid dissemination within the peritoneal cavity. ${ }^{66,67}$ The histology is similar to signet ring cell adenocarcinoma at other sites in the GI tract. They are composed of single cells, clusters, and/or sheets of signet ring cells, some with extracellular mucin. Tumors that have areas of goblet cell carcinoid may represent progression of a goblet cell carcinoid, and may be biologically distinct from pure signet ring cell carcinomas. ${ }^{11}$

\section{Differential Diagnosis}

Distinguishing mucinous adenocarcinoma from LAMN is based on evaluation of the manner of invasion and on the cytologic features of the neoplastic epithelium. LAMNs show prominent fibrosis of the wall with pushing invasion. Mucinous adenocarcinomas usually show conventional patterns of invasion exhibiting irregular pools of mucin harboring high-grade epithelium in complex architectural configurations, irregular shaped glands with a desmoplastic stromal response, or single cell invasion. By definition, LAMNs are low-grade tumors, whereas mucinous adenocarcinomas may be low or high grade.

Adenocarcinoma must also be distinguished from goblet cell carcinoid, a distinctive tumor of the appendix composed of cohesive nests of goblet-like

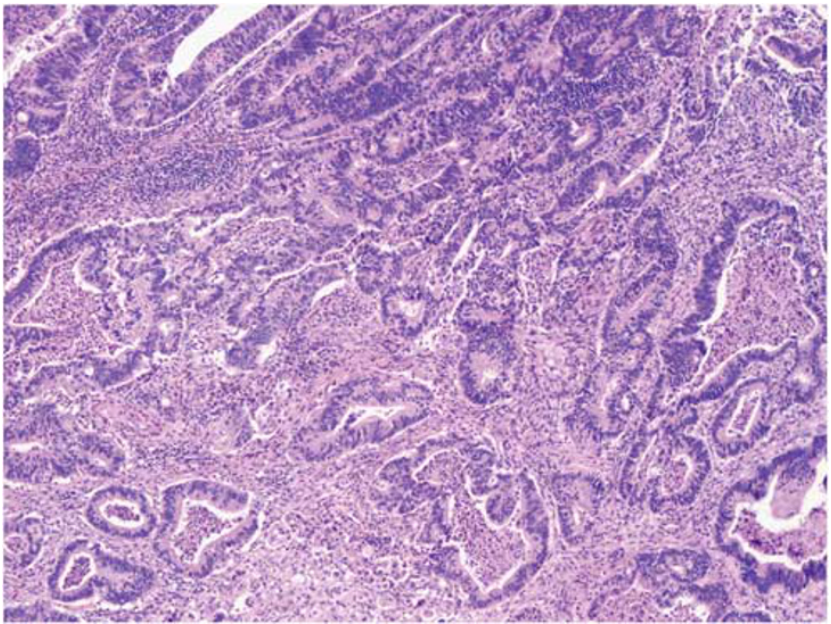

Figure 11 Invasive adenocarcinoma of the appendix. In this example, the invasive tumor has a colorectal appearance, with glands lined by pseudostratified, hyperchromatic columnar malignant cells and dirty necrosis within the glands.

cells admixed with endocrine cells, Paneth cells, and cuboidal glandular cells, with or without extracellular mucin. The orderly arrangement of the tumor cell clusters, circumferential growth pattern, and often low-grade cytologic features help to distinguish this group of tumors. Immunohistochemistry for neuroendocrine differentiation may be misleading in this differential since the presence of neuroendocrine differentiation may be focal in goblet cell carcinoid, and conversely may be prominent in conventional adenocarcinomas. Finally, appendiceal adenocarcinoma must be distinguished from metastasis to the appendix. Metastatic tumors can mimic primary tumors of the appendix both by their morphologic similarity to a primary adenocarcinoma and their extension into the mucosa that can be mistaken for a precursor of the tumor.

\section{Natural History}

The reported 5-year survival rates for patients with appendiceal adenocarcinoma ranges from 18.7\% (ref. 48 ) to $55 \% .{ }^{57}$ Patients with mucinous adenocarcinomas have a better prognosis than those with non-mucinous adenocarcinomas. ${ }^{57,58,62}$ Several studies have shown that histologic grade $8,49,61$ and Duke's stage correlate with prognosis. $8,57,58,61,62,68,69$ Patients with peritoneal carcinomatosis have a poor prognosis. ${ }^{10,52,60,62}$

\section{Treatment}

Invasive adenocarcinoma of the appendix warrants treatment by right hemicolectomy, with lymph node dissection, in order to stage the tumor and ensure complete resection..$^{57,59,62,68}$ Some authorities 
advocate oophorectomy in women, both for staging purposes and in order to remove a frequent site of metastasis. ${ }^{57,62}$ Treatment of patients with peritoneal spread depends on the resectability of the tumor. Patients whose tumors are resectable should be treated aggressively with peritonectomy and post-operative intraperitoneal chemotherapy (see PMP). ${ }^{46,70,71}$

\section{Pseudomyxoma peritonei}

PMP refers to the accumulation of mucin within the peritoneal cavity secondary to mucinous epithelial neoplasia (Figure 2). This most often occurs because of peritoneal spread of a mucinous neoplasm from the appendix but has been described with mucinous tumors from other sites, including colon, ovary, gallbladder, pancreas, and urachus.

\section{Clinical Features}

Spread of mucinous neoplastic epithelium to the peritoneal cavity occurs most often in association with LAMN and mucinous adenocarcinoma. Approximately $20 \%$ of patients with a mucinous tumor of the appendix develop PMP. ${ }^{72}$ Patients with PMP typically present in the 6th-7th decades of life, and in some series, women predominate over men. ${ }^{72-74}$ Peritoneal mucinous deposits tend to accumulate in particular areas, such as the greater omentum, the undersurface of the right hemidiaphragm, pelvis, right retrohepatic space, left abdominal gutter, and the ligament of Treitz. ${ }^{75}$ This so-called 'redistribution' phenomenon can be explained by the hypothesis that tumor accumulates at anatomic locations where ascitic fluid is resorbed from the abdomen, and in areas where fluid pools in the abdomen. ${ }^{11}$

\section{Pathology, Nomenclature, and Outcome}

In general, the morphologic characteristics and, in particular, the grade of the neoplastic epithelium correlates with the grade and degree of 'invasiveness' of the primary appendiceal tumor. ${ }^{10}$ Classical PMP, which occurs in patients with LAMN, demonstrates abundant mucin, hyalinized, fibrotic stroma, and occasional strips of low-grade mucinous epithelium (Figures 12 and 13). Peritoneal mucinous tumors with high-grade cytologic atypia of the neoplastic epithelium and more complex architecture are usually associated with mucinous adenocarcinomas of the appendix or elsewhere (Figure 14).

Ronnett et $a l^{65}$ separated peritoneal mucinous tumors into two grades and offered a classification system to describe these grades. They proposed the term disseminated peritoneal adenomucinosis (DPAM) for 'non-invasive' mucinous implants con- taining scant strips of simple or focally proliferative epithelium with minimal-to-moderate atypia and no significant mitotic activity. In contrast, peritoneal tumors characterized by more abundant proliferative epithelium arranged as glands, nests, or individual cells were classified as peritoneal mucinous carcinomatosis (PMCA). Long-term survival was significantly better for patients with DPAM as opposed to those with PMCA, with 5- and 10-year survival rates of 75 and $68 \%$ for patients with DPAM

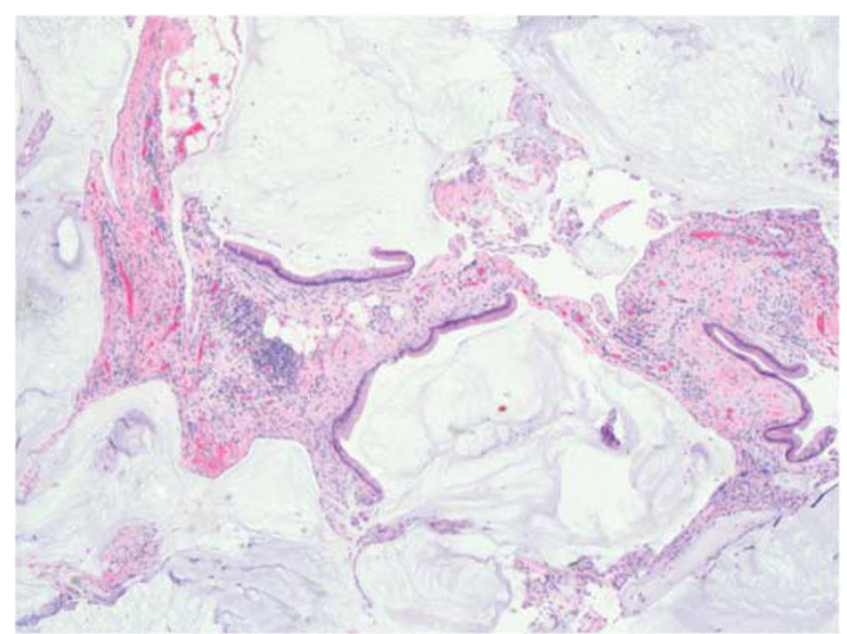

Figure 12 Low-grade peritoneal mucinous neoplasia. This peritoneal mucinous tumor shows classic features of pseudomyxoma peritonei, with abundant mucin and relatively scant mucinous epithelial groups arranged as strips with low-grade cytology. The terminology for low-grade pseudomyxoma peritonei is evolving, with international authorities favoring a diagnosis of mucinous adenocarcinoma peritonei, low grade, and discouraging the use of the term disseminated peritoneal adenomucinosis.

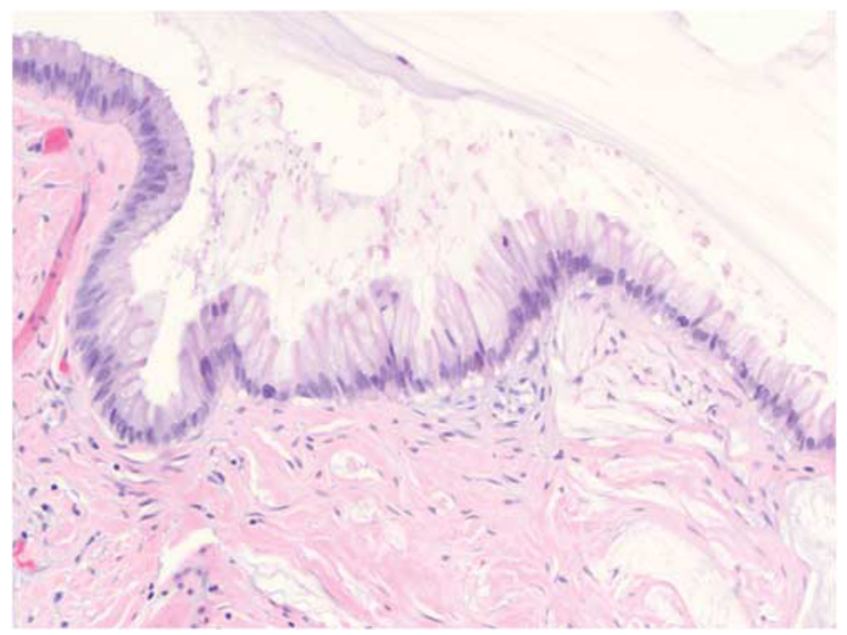

Figure 13 High power view of low-grade peritoneal mucinous neoplasia. The mucinous epithelium in the peritoneal mucinous tumor is remarkably bland with small nuclei. This incredibly benign nuclear morphology has contributed to the controversy over whether pseudomyxoma peritonei represents low-grade adenocarcinoma or peritoneal dissemination of adenomatous epithelium. 


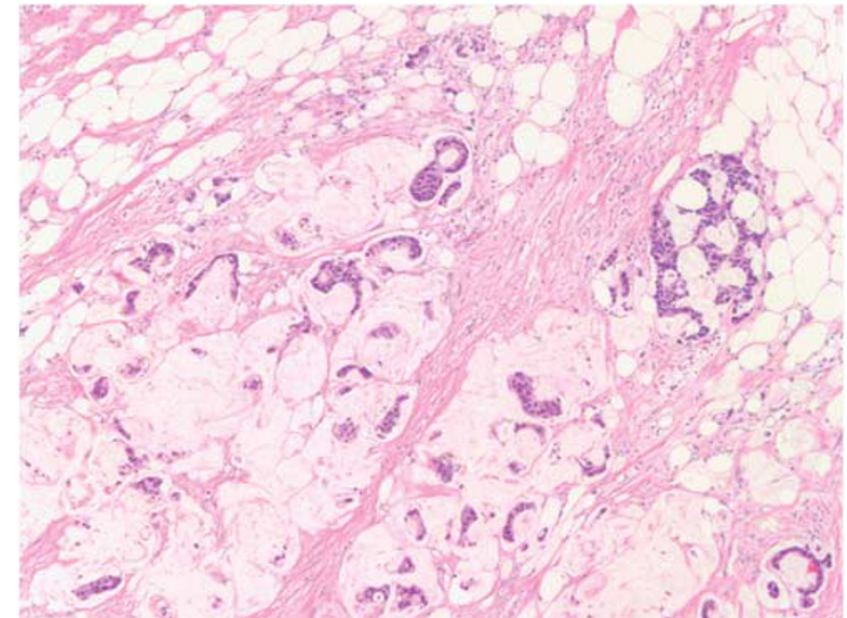

Figure 14 Mucinous adenocarcinoma peritonei, high grade. This peritoneal tumor has features that qualify as high grade, including high cellularity with malignant appearing nuclei, tumor budding, and desmoplastic tissue response.

and 14 and $3 \%$ for patients with PMCA. They also described an intermediate category for tumors that were mostly DPAM but with focal areas of welldifferentiated adenocarcinoma. ${ }^{65}$ Initially, these patients appeared to have an intermediate prognosis, but additional follow-up showed that they behaved like PMCA. ${ }^{76}$ In contrast, a study of 101 patients with PMP by Bradley et $a l^{77}$ found that patients with DPAM and intermediate grade peritoneal mucinous tumors had similar outcome, and that they fared better than those with high-grade peritoneal tumors. They argued that DPAM is in fact very well-differentiated mucinous carcinoma, and they proposed that all PMP be classified as either mucinous carcinoma peritonei, low grade, or mucinous carcinoma peritonei, high grade. More recently, Shetty et $a 7^{78}$ used a three-tiered grading system for PMP. In their system, PMP1 was analogous to DPAM; tumors with any amount of signet ring cell morphology were classified as PMP3 (Figure 15); and PMP2 was reserved for tumors that did not meet criteria for PMP1 or PMP3. Another study examining the influence of signet ring cells on prognosis $^{79}$ found that, among high-grade peritoneal mucinous adenocarcinomas from the appendix, the presence of signet ring cells had a negative impact on prognosis if the signet ring cells invaded tissue, but not if they were floating freely in the peritoneal mucin pools. These studies and others ${ }^{17,80}$ demonstrate that the grade of the peritoneal tumor predicts behavior of the tumor, independently of the grade of the primary tumor.

\section{Treatment}

The treatment for patients with PMP has not been standardized. Surgical tumor debulking was the

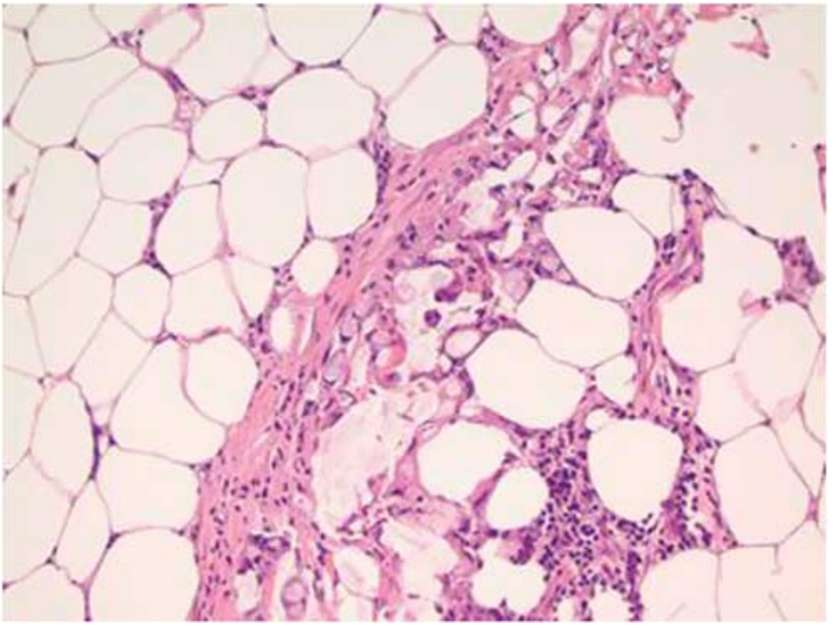

Figure 15 Signet ring cells in peritoneal mucinous neoplasia. Recent data suggest that the presence of signet ring cells in any amount may be a poor prognostic indicator in pseudomyxoma peritonei, and their presence qualifies the tumor as high grade in a two-tiered system or as pseudomyxoma peritonei-3 in a threetiered system.

mainstay of therapy for decades, but the majority of patients treated in that manner had disease recurrence that required repeated surgical debulking procedures until, eventually, adhesions precluded additional surgery and patients succumbed to their tumor. ${ }^{81,82}$ Survival rates for patients who underwent debulking were reported to be $86 \%, 53-67 \%$, and $32 \%$ at 2,5 , and 10 years, respectively. ${ }^{81,83}$ Sugarbaker and colleagues ${ }^{46,70,71,84}$ pioneered an aggressive treatment approach that involves peritonectomies in order to achieve complete cytoreduction and HIPEC. The cytoreduction procedure includes peritonectomies as well as resection of affected organs. Using this aggressive approach, they achieved 5- and 10-year survival rates of 71.9 and $54.5 \%{ }^{46}$ Other groups have reported similar survival rates using cytoreduction surgery and HIPEC. In particular, long-term survival is noted to be better in some studies. ${ }^{85-87}$ Several factors have been associated with success in the management of PMP. One of the most important factors is attaining complete cytoreduction, which is achieved in $40-91 \%$ of cases. ${ }^{87,88}$ In one study, patients who had complete cytoreduction had a 5 - and a 10 -year survival rate of $87 \%$ and $74 \%$, respectively, whereas those in which complete cytoreduction could not be achieved had $34 \%$ and $23 \% 5$ - and 10-year survival rates. ${ }^{87}$ In this regard, the experience of the surgeon may have a role, with specialized centers achieving better survival statistics. ${ }^{85,86}$ Extensive involvement of the small bowel or mesentery predicts a high likelihood of incomplete cytoreduction. As previously discussed, peritoneal tumor grade is another factor that affects outcome. ${ }^{85,86}$ 


\section{Future Directions in Appendiceal Tumor Classification}

Despite the progress that has been made in the classification of appendiceal mucinous neoplasia, there is still considerable variability in applying the WHO classification system. Even among experts, there are disagreements about whether LAMN is an appropriate diagnostic term in certain circumstances or, indeed, ever. A related question is whether to call a tumor with pushing invasion but high-grade cytology an adenocarcinoma or perhaps adopt HAMN, for high-grade appendiceal mucinous neoplasm. The relationship of serrated lesions of the appendix and goblet cell carcinoids to mucinous epithelial neoplasms awaits additional morphologic and molecular studies. In the area of PMP, there is still insufficient data to determine whether a 2- or 3 -tiered system is more prognostically meaningful. The criteria for classifying a peritoneal tumor as high grade and the importance of any amount of signet ring cell morphology in prognosis are additional areas of uncertainty. Recently, an international group of authorities on appendiceal neoplasia from a variety of medical specialties has begun the process of defining consensus criteria and nomenclature for these tumors under the auspices of the Peritoneal Surface Oncology Group International. By working toward uniform nomenclature and consensus criteria, groups such as this one will hopefully lay the groundwork for a consensus approach to appendiceal neoplasia that can be used to interpret molecular and genetic studies of these fascinating and enigmatic tumors.

\section{Disclosure/conflict of interest}

The authors declare no conflict of interest.

\section{References}

1 Werth R. Klinische und anatomische untersuchungen zur lehre von den bauchgeschwuelsten und der lapartomie. Arch f Gynaek 1884;24:100-118.

2 Fraenkel K. Uber das sogenannte pseudomyxoma peritonei. Muenchen med Wehnschr 1901;68:965-970.

3 Young RH. Pseudomyxoma peritonei and selected other aspects of the spread of appendiceal neoplasms. Semin Diagn Pathol 2004;21:134-150.

4 Woodruff R, McDonald JR. Benign and malignant cystic tumors of the appendix. Surg Gynecol Obstet 1940;71:750-755.

5 Qizilbash AH. Mucoceles of the appendix. Their relationship to hyperplastic polyps, mucinous cystadenomas, and cystadenocarcinomas. Arch Pathol Lab Med 1975;99:548-555.

6 Parsons J, Gray GF, Thorbjarnarson B. Pseudomyxoma peritonei. Arch Surg 1970;101:545-549.

7 Wackym PA, Gray GF Jr. Tumors of the appendix: I. Neoplastic and nonneoplastic mucoceles. South Med J 1984;77:283-287.
8 Wolff M, Ahmed N. Epithelial neoplasms of the vermiform appendix (exclusive of carcinoid). I. Adenocarcinoma of the appendix. Cancer 1976;37: 2493-2510.

9 Wolff M, Ahmed N. Epithelial neoplasms of the vermiform appendix (exclusive of carcinoid). II. Cystadenomas, papillary adenomas, and adenomatous polyps of the appendix. Cancer 1976;37:2511-2522.

10 Ronnett BM, Kurman RJ, Zahn CM, et al. Pseudomyxoma peritonei in women: a clinicopathologic analysis of 30 cases with emphasis on site of origin, prognosis, and relationship to ovarian mucinous tumors of low malignant potential. Hum Pathol 1995;26:509-524.

11 Carr NJ, Sobin LH. Unusual tumors of the appendix and pseudomyxoma peritonei. Semin Diagn Pathol 1996;13:314-325.

12 Higa E, Rosai J, Pizzimbono CA, et al. Mucosal hyperplasia, mucinous cystadenoma, and mucinous cystadenocarcinoma of the appendix. A re-evaluation of appendiceal 'mucocele'. Cancer 1973;32:1525-1541.

13 Bradley RF, Cortina G, Geisinger KR. Pseudomyxoma peritonei: Review of the controversy. Curr Diagn Pathol 2007;13:410-416.

14 Carr NJ, Sobin LH. Adenocarcinoma of the appendix, In: Bosman FT, Carneiro F, Hruban RH, et al. (eds) World Health Organization Classification of Tumours of the Digestive System. IARC Press: Lyon, France; 2010, pp 122-125.

15 Edge SB, Byrd DR, Compton CC, et al. (eds). AJCC Cancer Staging Manual. (Appendix) Springer: New York, USA; 2010, pp 133-141.

16 Carr NJ, McCarthy WF, Sobin LH. Epithelial noncarcinoid tumors and tumor-like lesions of the appendix. A clinicopathologic study of 184 patients with a multivariate analysis of prognostic factors. Cancer 1995;75:757-768.

17 Misdraji J, Yantiss RK, Graeme-Cook FM, et al. Appendiceal mucinous neoplasms: a clinicopathologic analysis of 107 cases. Am J Surg Pathol 2003; 27:1089-1103.

18 Pai RK, Beck AH, Norton JA, et al. Appendiceal mucinous neoplasms: clinicopathologic study of 116 cases with analysis of factors predicting recurrence. Am J Surg Pathol 2009;33:1425-1439.

19 Williams GR, du Boulay CE, Roche WR. Benign epithelial neoplasms of the appendix: classification and clinical associations. Histopathology 1992;21: 447-451.

20 Rudek B, von Herbay A, Schmidt J. Intussusception of the appendix secondary to mucinous cystadenoma. Dig Surg 2001;18:422-426.

21 Jones CD, Eller DJ, Coates TL. Mucinous cystadenoma of the appendix causing intussusception in an adult. Am J Gastroenterol 1997;92:898-899.

22 Heithold DL, Tucker JG, Lucas GW. Appendiceal intussusception as a manifestation of mucinous cystadenoma of the appendix: an interesting clinical entity. Am Surg 1997;63:390-391.

23 Chetty R, Daniel WJ. Mucinous cystadenoma of the appendix: an unusual cause of recurrent intussusception in an adult. Aust N Z J Surg 1992;62:670-671.

24 Ashrafi M, Joshi V, Zammit M, et al. Intussusception of the appendix secondary to mucinous cystadenoma: A rare cause of abdominal pain. Int J Surg Case Rep 2011;2:26-27.

25 Cois A, Pisanu A, Pilloni L, et al. Intussusception of the appendix by mucinous cystadenoma. Report of a 
case with an unusual clinical presentation. Chir Ital 2006;58:101-104.

26 Casey RG, Ofori-Kuma F, Carroll K, et al. Intussusception of the appendix by a villous adenoma. Eur J Surg 2001;167:789-791.

27 Tonsekar KS, Cacdac R, Ashare R, et al. Villous adenoma of the vermiform appendix with cecal intussusception: a case report and review of literature. Am Surg 1994;60:982-984.

28 Sadahiro S, Ohmura T, Yamada Y, et al. A case of cecocolic intussusception with complete invagination and intussusception of the appendix with villous adenoma. Dis Colon Rectum 1991;34:85-88.

29 Collins DC. Adenomatous polyps of the vermiform appendix. Surg Clin North Am 1932;12:1063-1067.

30 Brown WG, Van Santen E. Carcinoma in situ in adenomatous polyps of vermiform appendix. Tex Med 1970;66:52-53.

31 Appelman HD. Epithelial neoplasia of the appendix, In: Norris H (ed). Pathology of the Colon, Small Intestine, and Anus. Churchill Livingstone: New York, USA; 1991, pp 263-303.

32 Young RH, Gilks CB, Scully RE. Mucinous tumors of the appendix associated with mucinous tumors of the ovary and pseudomyxoma peritonei. A clinicopathological analysis of 22 cases supporting an origin in the appendix. Am J Surg Pathol 1991;15:415-429.

33 Chen KT, Davies OG. Mucinous cystadenoma of the appendix appearing as an inguinal hernia. Arch Pathol Lab Med 1982;106:423-424.

34 Young RH, Rosenberg AE, Clement PB. Mucin deposits within inguinal hernia sacs: a presenting finding of low-grade mucinous cystic tumors of the appendix. A report of two cases and a review of the literature. Mod Pathol 1997;10:1228-1232.

35 Esquivel J, Sugarbaker PH. Pseudomyxoma peritonei in a hernia sac: analysis of 20 patients in whom mucoid fluid was found during a hernia repair. Eur J Surg Oncol 2001;27:54-58.

36 Prayson RA, Hart WR, Petras RE. Pseudomyxoma peritonei. A clinicopathologic study of 19 cases with emphasis on site of origin and nature of associated ovarian tumors. Am J Surg Pathol 1994;18:591-603.

37 Hsu M, Young RH, Misdraji J. Ruptured appendiceal diverticula mimicking low-grade appendiceal mucinous neoplasms. Am J Surg Pathol 2009;33:1515-1521.

38 Stockl T, Ross JS, Walter O, et al. Appendiceal mucosal Schwann cell proliferation: a putative histologic marker of appendiceal diverticular disease. Int J Surg Pathol 2013;21:603-609.

39 Mitchell A, Dube P, Sideris L. Dysplastic intestinaltype metaplasia of appendiceal endometriosis: a mimic of low grade appendiceal mucinous neoplasm. Diagn Pathol 2014;9:39.

40 Mai KT, Burns BF. Development of dysplastic mucinous epithelium from endometriosis of the appendix. Histopathology 1999;35:368-372.

41 Tipps AM, Weidner N. Colonization of intestinal endometriosis by benign colonic mucosa: a pattern potentially misdiagnosed as invasive mucinous carcinoma. Int J Surg Pathol 2011;19:259-262.

42 Libbrecht L, Snauwaert C, De Vos M, et al. Intestinal metaplasia and colonization of endometriosis in a case of an appendiceal mucinous neoplasm. Virchows Arch 2012;461:227-229.

43 Misdraji J, Lauwers GY, Irving JA, et al. Appendiceal or cecal endometriosis with intestinal metaplasia: a potential mimic of appendiceal mucinous neoplasms. Am J Surg Pathol 2014;38:698-705.

44 Yantiss RK, Shia J, Klimstra DS, et al. Prognostic significance of localized extra-appendiceal mucin deposition in appendiceal mucinous neoplasms. Am J Surg Pathol 2009;33:248-255.

45 Arnason T, Kamionek M, Yang M, et al. Significance of proximal margin involvement in low grade appendiceal mucinous neoplasms. Arch Pathol Lab Med 2014; (in press).

46 Gonzalez-Moreno S, Sugarbaker PH. Right hemicolectomy does not confer a survival advantage in patients with mucinous carcinoma of the appendix and peritoneal seeding. Br J Surg 2004;91:304-311.

47 Foster JM, Gupta PK, Carreau JH, et al. Right hemicolectomy is not routinely indicated in pseudomyxoma peritonei. Am Surg 2012;78:171-177.

48 Collins DC. A study of 50000 specimens of the human vermiform appendix. Surg Gynecol Obstet 1955;101: 437-446.

49 Chang P, Attiyeh FF. Adenocarcinoma of the appendix. Dis Colon Rectum 1981;24:176-180.

50 Andersson A, Bergdahl L, Boquist L. Primary carcinoma of the appendix. Ann Surg 1976;183:53-57.

51 Flint FB, Kahn AM, Passaro E Jr. Adenocarcinoma of the appendix. Am J Surg 1970;120:707-709.

52 Cerame MA. A 25-year review of adenocarcinoma of the appendix. A frequently perforating carcinoma. Dis Colon Rectum 1988;31:145-150.

53 McGregor JK, McGregor DD. Adenocarcinoma of the appendix. Surgery 1960;48:925-935.

54 Hopkins GB, Tullis RH, Kristensen KA. Primary adenocarcinoma of the vermiform appendix: report of seven cases and review of the literature. Dis Colon Rectum 1973;16:140-144.

55 Sieracki JC, Tesluk H. Primary adenocarcinoma of the vermiform appendix. Cancer 1956;9:997-1011.

56 Harris GJ, Urdaneta LF, Mitros FA. Adenocarcinoma of the vermiform appendix. J Surg Oncol 1990;44:218-224.

57 Nitecki SS, Wolff BG, Schlinkert R, et al. The natural history of surgically treated primary adenocarcinoma of the appendix. Ann Surg 1994;219:51-57.

58 Didolkar MS, Fanous N. Adenocarcinoma of the appendix: a clinicopathologic study. Dis Colon Rectum 1977;20:130-134.

59 Conte CC, Petrelli NJ, Stulc J, et al. Adenocarcinoma of the appendix. Surg Gynecol Obstet 1988;166:451-453.

60 Proulx GM, Willett CG, Daley W, et al. Appendiceal carcinoma: patterns of failure following surgery and implications for adjuvant therapy. J Surg Oncol 1997;66:51-53.

61 Gilhome RW, Johnston DH, Clark J, et al. Primary adenocarcinoma of the vermiform appendix: report of a series of ten cases, and review of the literature. $\mathrm{Br} \mathrm{J}$ Surg 1984;71:553-555.

62 Cortina R, McCormick J, Kolm P, et al. Management and prognosis of adenocarcinoma of the appendix. Dis Colon Rectum 1995;38:848-852.

63 Uihlein A, McDonald JR. Primary carcinoma of the appendix resembling carcinoma of the colon. Surg Gynecol Obstet 1943;76:711-714.

64 Kabbani W, Houlihan PS, Luthra R, et al. Mucinous and nonmucinous appendiceal adenocarcinomas: different clinicopathological features but similar genetic alterations. Mod Pathol 2002;15:599-605.

65 Ronnett BM, Zahn CM, Kurman RJ, et al. Disseminated peritoneal adenomucinosis and peritoneal mucinous carcinomatosis. A clinicopathologic analysis of 109 
cases with emphasis on distinguishing pathologic features, site of origin, prognosis, and relationship to 'pseudomyxoma peritonei'. Am J Surg Pathol 1995;19: 1390-1408.

66 Qizilbash AH. Primary adenocarcinoma of the appendix. A clinicopathological study of 11 cases. Arch Pathol Lab Med 1975;99:556-562.

67 McCusker ME, Cote TR, Clegg LX, et al. Primary malignant neoplasms of the appendix: a population-based study from the surveillance, epidemiology and endresults program, 1973-1998. Cancer 2002;94:3307-3312.

68 Ferro M, Anthony PP. Adenocarcinoma of the appendix. Dis Colon Rectum 1985;28:457-459.

69 Lenriot JP, Huguier M. Adenocarcinoma of the appendix. Am J Surg 1988;155:470-475.

70 Sugarbaker PH, Jablonski KA. Prognostic features of 51 colorectal and 130 appendiceal cancer patients with peritoneal carcinomatosis treated by cytoreductive surgery and intraperitoneal chemotherapy. Ann Surg 1995;221:124-132.

71 Sugarbaker PH, Zhu BW, Sese GB, et al. Peritoneal carcinomatosis from appendiceal cancer: results in 69 patients treated by cytoreductive surgery and intraperitoneal chemotherapy. Dis Colon Rectum 1993;36: 323-329.

72 Smeenk RM, van Velthuysen ML, Verwaal VJ, et al. Appendiceal neoplasms and pseudomyxoma peritonei: a population based study. Eur J Surg Oncol 2008;34:196-201.

73 Costa MJ. Pseudomyxoma peritonei. Histologic predictors of patient survival. Arch Pathol Lab Med 1994;118:1215-1219.

74 Fernandez RN, Daly JM. Pseudomyxoma peritonei. Arch Surg 1980;115:409-414.

75 Sugarbaker PH. Pseudomyxoma peritonei. A cancer whose biology is characterized by a redistribution phenomenon. Ann Surg 1994;219:109-111.

76 Ronnett BM, Yan H, Kurman RJ, et al. Patients with pseudomyxoma peritonei associated with disseminated peritoneal adenomucinosis have a significantly more favorable prognosis than patients with peritoneal mucinous carcinomatosis. Cancer 2001;92:85-91.

77 Bradley RF, JHt Stewart, Russell GB, et al. Pseudomyxoma peritonei of appendiceal origin: a clinicopathologic analysis of 101 patients uniformly treated at a single institution, with literature review. Am J Surg Pathol 2006;30:551-559.
78 Shetty S, Natarajan B, Thomas P, et al. Proposed classification of pseudomyxoma peritonei: influence of signet ring cells on survival. Am Surg 2013;79: 1171-1176.

79 Sirintrapun SJ, Blackham AU, Russell G, et al. Significance of signet ring cells in high-grade mucinous adenocarcinoma of the peritoneum from appendiceal origin. Hum Pathol 2014;45:1597-1604.

80 Carr NJ, Finch J, Ilesley IC, et al. Pathology and prognosis in pseudomyxoma peritonei: a review of 274 cases. J Clin Pathol 2012;65:919-923.

81 Gough DB, Donohue JH, Schutt AJ, et al. Pseudomyxoma peritonei. Long-term patient survival with an aggressive regional approach. Ann Surg 1994;219: 112-119.

82 Miner TJ, Shia J, Jaques DP, et al. Long-term survival following treatment of pseudomyxoma peritonei: an analysis of surgical therapy. Ann Surg 2005;241: 300-308.

83 Jarvinen P, Jarvinen HJ, Lepisto A. Survival of patients with pseudomyxoma peritonei treated by serial debulking. Colorectal Dis 2010;12:868-872.

84 Sugarbaker PH, Chang D. Results of treatment of 385 patients with peritoneal surface spread of appendiceal malignancy. Ann Surg Oncol 1999;6:727-731.

85 Elias D, Gilly F, Quenet F, et al. Pseudomyxoma peritonei: a French multicentric study of 301 patients treated with cytoreductive surgery and intraperitoneal chemotherapy. Eur J Surg Oncol 2010;36:456-462.

86 Smeenk RM, Verwaal VJ, Antonini N, et al. Survival analysis of pseudomyxoma peritonei patients treated by cytoreductive surgery and hyperthermic intraperitoneal chemotherapy. Ann Surg 2007;245:104-109.

87 Youssef H, Newman C, Chandrakumaran K, et al. Operative findings, early complications, and long-term survival in 456 patients with pseudomyxoma peritonei syndrome of appendiceal origin. Dis Colon Rectum 2011;54:293-299.

88 Moran B, Baratti D, Yan TD, et al. Consensus statement on the loco-regional treatment of appendiceal mucinous neoplasms with peritoneal dissemination (pseudomyxoma peritonei). J Surg Oncol 2008;98: 277-282.

89 Misdraji J. Epithelial neoplasms of the appendix. In: Odze RD, Goldblum JR (eds). Surgical Pathology of the GI Tract, Liver, Biliary tract, and Pancreas. Elsevier: Philadelphia, USA, 2014. 